\title{
The Principles of TCM Noun Terms Translation based on the Characteristics of TCM Language and Culture *
}

\author{
Dian-Gang LIU ${ }^{1, a}$, He-Rong MAO ${ }^{1, b^{*}}$ and Chi GU ${ }^{1, c}$ \\ ${ }^{1}$ Center for Compilation, Translation and International Communication of Jingchu TCM Literature, \\ School of Foreign Languages, Hubei University of TCM, No. 1, West Huang Jiahu Road, Hongshan \\ District, Wuhan City, Hubei Province, 430065, PRC. \\ a liudiangang@163.com , trans_morning123@126.com, ${ }^{c}$ guchihbtcm@163.com \\ *Herong MAO, Center for Compilation, Translation and International Communication of Jingchu TCM \\ Literature, School of Foreign Languages, Hubei University of TCM, No. 1, West Huang Jiahu Road, \\ Hongshan District, Wuhan City, Hubei Province, 430065, PRC. \\ Telephone: +86-13986187098, E-mail: trans_morning123@126.com
}

Keywords: Characteristics of TCM language and culture; TCM noun terms; English translation; principles

\begin{abstract}
As TCM is being spread to a wider world, TCM translation from Chinese to other languages especially to English is becoming increasingly important. Due to the fact that most basic TCM noun terms are closely connected with the unique characteristics of TCM language and culture, like being embedded with philosophical speculation, frequent use of literary metaphorical techniques, highly generalization of the fundamental theories, it's typically necessary to rethink about the principles that can be used in TCM translation. In this paper, we propose to translate out the medical connotations to show the scientific nature of TCM, to translate with modern medical terms while trying hard to maintain its Chinese characteristics and translate from the exterior to the interior to better communicate TCM.
\end{abstract}

\section{Introduction}

Language is so closely related to the culture where it's conceived. It's a generally acknowledged fact that the language is the carrier of a culture, for how the culture is expressed and sustained relies heavily on the culture, while the culture exists as the mother of the language, where it will be powerless and obscure without the culture it comes from. The prominent Russian literary scholar, semiotician, and cultural historian Yuri Mikhailovich Lotman once pointed out, there isn't any language that is not deeply rooted in some kind of concrete culture and there isn't any culture that is centered around some kind of linguistic structure.[1] So do traditional Chinese medicine(TCM) culture and the unique language system that TCM uses. TCM, as a distinctive medical system compared with western medicine, has originated from the ancient Chinese philosophy, literature and knowledge in other fields. In the long history of development, TCM has formed its own language system, dripping with an obvious demonstration of philosophical speculation, metaphorical techniques in literature, highly generalization and gradual evolution of terms. Being characterized with such a feature, TCM cannot escape from some intricate problems in its Chinese-English translation, especially for quite some of its noun terms. Such challenges for sure will set barriers on the road of international communication of TCM culture.

\section{Characteristics of TCM Language}

\section{Being Embedded with Philosophical Speculation}

This research is financially supported by academic program of Hubei provincial department of education (15Q112). 
Based on the ancient Chinese philosophical thoughts, the fundamental theories and clinical practice of TCM visibly reflect the light of philosophical speculation. Holism and treatment based on syndrome differentiation are generally believed to be the two pillars of TCM's fundamental theories. Holism takes the different parts of human body as an organic whole, and the natural and societal factors all exert some influence on the health conditions of human being, like people's will, desire and emotions, even the family they are from and the occupations they go in for, which are totally unbelievable for western medicine, who holds that if there is any problem with the leg, then the leg should be treated. The unique concepts of TCM are highly consistent with the traditional Chinese philosophical thoughts such as the Unity of Mankind and Nature. The theories of Yin-Yang, Five-phases, Essential Qi, Visceral Manifestation etc., they are all from and vivid embodiment of the simple materialist dialectics of the ancient Chinese philosophy. This is quite difficult for modern Chinese people, not to mention people from other countries without sound understanding of traditional Chinese culture. So it's self-evident that problems arise when we are trying to translate TCM texts from Chinese to English, for both the translators from the source language and readers from the targeted language often cannot find equivalents for many words and phrases. [2]

\section{Use of Literary Metaphorical Techniques}

Analogy in the first place, is a writing technique employed in the literary works, while TCM uses a lot of analogical thoughts in its discourse system for different purposes. For euphemistic purpose, TCM designates gynecologists as doctors for gynaecological diseases; the blood chamber as a woman's womb, the house for storing blood; obstruction of a tunnel as menopause, so on and so forth, partly because traditionally Chinese people find it embarrassing talking about sex, anything related to sex in public. To make a point more concise and vivid, TCM uses quite a few fixed expressions, like sailing against the current(ni shui xing zhou), which really wants to express the efforts TCM practitioners make to dispel the exterior pathogens and induce dieresis by administrating the right medicines. For another example, broken metal won't ring(jin po bu ming) actually means hoarseness due to the deficiency of the Lung Qi often because of a chronic disease. And such terms are expressing some ideas with characteristics of TCM culture in the metaphorical way. In a word, TCM often compares the familiar with the unfamiliar, the known with the unknown, the simple with the complicated, the concrete with the abstract, which a palpable feature of the language that TCM uses. [3]

\section{Highly Generalization of the Fundamental Theories}

TCM language system is highly generalized, which is especially clearly seen in its fundamental theories. Take Yin-Yang Theory for example. What's Yin? What's Yang? They don't mean some objective things, but an endless group of things - things with the properties of water(like what moistens, flows downward) belong to Yin, while things with the properties of fire (like what lights, moves upward)belong to Yang. Because Yin and Yang are infinite and rich in connotations, we have to reach out to the Chinese pinyin in translating them into English. Another example is four medical examinations in TCM. How should wen be translated into English? Wen means both smelling with nose and hearing with ears. So we'd better translate the single Chinese word into two English words, smelling and listening, or auscultation and olfaction more professionally. TCM's special language system poses many threats to translators as well as readers.

\section{Principles of TCM Noun Terms Translation}

The characteristics of TCM language and culture determines that when we are translating TCM texts from Chinese to English, we have to employ some principles in treating TCM terms especially TCM Noun terms. 


\section{Translating out the Medical Connotation to Show the Scientific Nature of TCM}

Though TCM is so deeply rooted in and influenced by traditional Chinese culture, whose language system is permeated with traditional Chinese philosophical speculation and metaphors in literature, and whose fundamental theories are so highly generalized, it's after all a medicine and has been playing an indispensable role in the survival and prosperity of Chinese peoples. So in the translation of TCM texts, we can absorb nutrients and learn great lessons from how western medicine was introduced to China at the very beginning. [4] In other words, in doing translation, the medical meanings of most basic TCM noun terms should be translated out; TCM translation on the whole is translation of science and medicine rather than philosophy and literature. [5] We can learn a lot from the words of an extremely respected Chinese scientist Qian Xuesen, who once commented that there is only one road to the development of TCM, that is, TCM should be equipped with the strong modern science system, should be released from the shackles of the statements of ancient Chinese natural philosophy and philosophical speculation, and TCM should express itself with modern language system. [6]

\section{Translating with Modern Medical Terms While Trying Hard to Maintain Its Chinese Characteristics}

TCM and modern medicine, though, have are quite different medical systems, they have more in common than the differences. In this sense, in TCM translation, we can and have to learn as much as possible from modern medicine, especially the translation of most noun terms. After all, they can and have reached a wide consensus on the understanding of human body structure, the physiological functions, pathological conditions and treatments etc.

However, it doesn't mean that TCM translation should be westernized, that we should try every means to discover the equivalents of western medicine for all TCM terms. That's quite harmful and impossible. As the Chinese scholar Xun Chongxin once pointed out, there are quite different ideological foundations, values and outlooks for different cultures, therefore, in translation from one culture to another, cultural coagulationn may occur if we have to infuse the blood of a heterogeneous culture into its own, or vice versa. Take the human organs for example. We really know that the five Zang organs, Liver, Heart, Spleen, Lung, Spleen in TCM are not the same things in western medicine or modern medicine. But how can we create a new set of terms in English? So often we just take the terms from western medicine, but to show the differences, we may add an explanation or note right after, like Xin is frequently translated as Heart (a functional system in TCM, which is the monarch of the whole body and governs human blood vessels and spirit). However, for terms Yin, Yang, Qi, Jing, Sanjiao, we tend to translate them into Chinese Pinyin, for there are no such concepts in western culture, and it's impossible to find out the not-so-bad equivalents in western medicine. In fact, we are trying hard to maintain the Chinese characteristics of TCM when we are translating the typically Chinese concepts this way.

\section{Translating from the Exterior to the Interior to Better Communicate TCM}

As mentioned above, TCM language is a high-context language, to better communicate TCM to the world, we have to translate most TCM terms into something with concrete images. Only in this way can we bridge the gap caused by cultural deficit as much as possible and get TCM more widely recognized and accepted by those who are not familiar with TCM and traditional Chinese culture. As the example mentioned above, how to make foreigners know what's jin po bu ming? If we translate it into broken metal doesn't ring, it will definitely get readers confused, not to speak of another version, broken gold doesn't ring. Briefly, it means hoarseness or aphonia due to deficiency of the Lung Qi. Therefore, tu bu zhi shui should not be directly translated into earth failing to control water, but fluid metabolism disorder due to deficiency of the spleen for better understanding for foreigners. Then, if they become interested in TCM, they may find by their own efforts the embedded Five-phase theory in TCM culture. 


\section{Conclusion}

Traditional Chinese medicine is a high epitome of traditional Chinese culture, whose language system is quite different from that of modern medicine, or even today's language system Chinese people are using, especially for some of the highly-generalized noun terms. So, in TCM translation, we have to employ relevant principles, like translating out the medical meaning to show the scientific nature of TCM, translating with modern medical terms while trying hard to maintain its Chinese characteristics and translating from the exterior to the interior to better communicate TCM. What's the most important, as we always believe, is the accurate understanding of the terms and texts themselves. As the examples mentioned above, di dao bu tong, jin po bu ming etc., the vulgar or even wrong translation of TCM noun terms often stem from inaccurate or even wrong understandings. In this sense, both the translators and readers should work hard to learn more about TCM and traditional Chinese culture.

\section{Acknowledgments}

This research is financially supported by academic program of Hubei provincial department of education (15Q112). Sincere thanks also go to colleagues from the Center for Compilation, Translation and International Communication of Jingchu TCM Literature for their constructive suggestions in the writing of the paper.

\section{References}

[1] Guo Jianzhong. Cultural Elements in Translation: Foreignization and Domestication [J]. Journal of Foreign Languages,1998,7(2):12-18.

[2] Zhang Xiaoming. Research on English Translation of TCM[J]. Asia-Pacific Traditional Medicine, 2009,5(9):180.

[3] Min Shuyao. On the Translation of Metaphors in TCM from the Angle of Perception[J].Journal of Liaoning University of Chinese Medicine,2008,10(8):175.

[4] Liu Ya, Liang Zhong. Research on the Translational History Development and Influence of the Introduction of Western Medicine to China in the Ming and Qing Dynasties[J]. Hubei Social Sciences, 2015, (09):121-125.

[5] Li Yong'an. On How to Deal with the Cultural Elements in TCM Translation[J]. Chinese Journal of Integrated Traditional and Western Medicine,2002,22(6):468.

[6] Cao Shanying. On Translation of TCM Culture[J]. Journal of Chinese Science and Technology,2002,15(2),13.

[7] Xu Chognxin. Cultural Communication and Translation[J].Foreign Languages, 1991,1(71):32. 